\title{
Phytoprotection
}

\section{Index des sujets, volume 83}

Subject Index, Volume 83

Volume 83, numéro 3, 2002

URI : https://id.erudit.org/iderudit/706241ar

DOI : https://doi.org/10.7202/706241ar

Aller au sommaire du numéro

\section{Éditeur(s)}

Société de protection des plantes du Québec (SPPQ)l

\section{ISSN}

0031-9511 (imprimé)

1710-1603 (numérique)

Découvrir la revue

Citer ce document

(2002). Index des sujets, volume 83. Phytoprotection, 83(3), 171-173.

https://doi.org/10.7202/706241ar d'utilisation que vous pouvez consulter en ligne.

https://apropos.erudit.org/fr/usagers/politique-dutilisation/ 


\section{Index des sujets, volume 83 Subject Index, Volume 83}

\section{A-B}

Accouplements multiples

Acer saccharum

Acrobasis vaccinii

Actinomycète / actinomycete

Activité enzymatique

Alberta

Aphids

Asparagus

Asperge

Azadirachta indica

Bacillus thuringiensis

Bacillus thuringiensis var. kurstaki

Bioclimatic models

Biodiversité / biodiversity

Bioessai / bioassay

Biological control

Blumeria graminis f. sp. hordei

Bourdons

Brassica juncea

Brassica rapa

"Bulk" method

Bumblebees

\section{C-D}

\section{Canneberge}

Cannibalisme / cannibalism

Canola / canola $31,61,161,162,163$

Carabidae

Ceratocystis resinifera

Ceutorhynchus obstrictus

Choristoneura fumiferana

Choristoneura rosaceana

Chrysoteuchia topiaria

Coleomegilla maculata

Colorado potato beetle

Compétition intra-spécifique

Concombre de serre

Cranberry

Cultivar susceptibility

Culture intercalaire

Cyathus olla

Damage potential

Décomposition du chaume

Defoliation / défoliation

159

$60,62,139$

158

31

63

160,166

160,166

$55,61,66$

$52,54,56,59$

60,167

139

55,63
Delia radicum 163

Désherbage localisé

161

89

Détection / detection 157

Doryphore de la pomme de terre 89

Doubled haploid

131

\section{E-H}

Entomofaune pyrophile $\quad 65$

Enzymatic activity $\quad 31$

Farnésyle diphosphate synthase $\quad 47$

Filiation unipare $\quad 131$

Foraging 63

Fumigation / fumigation $\quad 147$

Fusarium 160, 166

Fusarium oxysporum

f. sp. radicis-lycopersici 73

Genetic resistance $\quad 131$

Genetic variability $\quad 54$

Gliocladium virens $\quad 160$

GPS $\quad 161$

Greenhouse cucumber $\quad 73$

Greenhouse tomato $\quad 73$

Growth regulator $\quad 53$

Haplodiploïdisation $\quad 131$

Harmonia axyridis $\quad 55$

Helminthosporium solani 165

Hernie des crucifères $\quad 162$

Hordeum spontaneum $\quad 125$

Horizontal resistance $\quad 125$

Hormone juvénile $\quad 47$

Host quality $\quad 65$

Host selection $\quad 52$

Hyaliodes vitripennis $\quad 66$

I-L

Imazamox 99

Imazethapyr 99

Imidazolinone $\quad 99$

Insectes / insects $\quad 139$

Integrated control 49

Integrated crop management $\quad 49,67,69$

Integrated disease management

1

Integrated fruit production $53,57,58,64$

Intercropping

115

Intraguild predation

$55,60,64$ 
Intraspecific competition

Lambdina fiscellaria

Leptinotarsa decemlineata

Lesion nematode

Ligninolytic enzymes

Linum usitatissimum

Localised weed control

Locusta migratoria

Lutte biologique

Lutte intégrée

Lutte physique

Lygus lineolaris

\section{M-O}

Macrosiphom euphorbiae

Manduca sexta

Mayetiola destructor

Metarhizium anisopliae var. acridum 62

Méthode "bulk"

Migration / migration

Modèles bioclimatiques

Nasonovia ribisnigri

Nématode / nematode 41,66,115,147, 166

Nématode des lésions racinaires 111,158

Newfoundland

Nicosulfuron

Oïdium

Ophiostoma novo-ulmi

Ostrinia nubilalis

Overwintering survival

\section{P-Q}

Paratylenchus projectus

Pennisetum glaucum

Phenology

Physical control

Phytohormones

Phytophtora infestans

Picea abies

Pissodes strobi

Plasmodiophora brassicae

Plutella xylostella

Pollination

Pollinisation

Polygalacturonase

Pomme de terre

Potato

Potentiel membranaire

Pourriture des racines

Powdery mildew

Pratylenchus penetrans
60

48

89

115

31

147

161

62

$63,158,166$

1,49

67,164

164

56

52

131

131

68

51

68

\section{6}

139

99

125

164

48

89

147

158

89

67,164

159

158

62

62

162

61

52

52

31

$49,69,89$

$49,69,89$

54

157

125

$41,111,115,158$
Prédation intraguilde

$55,60,64$

Production fruitière intégrée $53,57,58,64$

Production intégrée

$49,67,69$

Pseudomonas chlororaphis

165

Pseudomonas putida

165

Pucerons

Pythium ultimum

Qualité de I'hôte

\section{R-S}

Régulateur de croissance 53

Release strategy

Résistance / resistance

Résistance génétique

$41,125,167$

131

Rhagoletis pomonella

Rhizoctonia solani 73

Root rot 157

Root-lesion nematode 111

Rotation / rotation 163

Rudbeckia 115

Sclerotinia sclerotiorum $\quad 73,160$

Sélection de l'hôte 52

Sensibilité variétale 1

Single seed descent $\quad 131$

Sorghum bicolor $\quad 147$

Soya $\quad 157,160$

Soybean

157,160

Sparganothis sulfureana $\quad 62$

Stratégie d'introduction $\quad 66$

Streptomyces griseus $\quad 165$

Streptomycètes / streptomycetes $\quad 165$

Stubble decomposition 31

Sulfonylurea 99

Suppression 115

Survie hivernale $\quad 89$

Syrphophagus aphidivorus $\quad 52$

\section{T-W}

Technique REMI 164

Telone 147

Terre-Neuve $\quad 139$

Tetranychus urticae. $\quad 66$

Tipulidae 66, 166

Tomate de serre 73

Tranosema rostrale $\quad 59$

Trichoderma harzianum 73

Trichogramma 50,62, 68

Trichogramma evanescens $\quad 57,65$

Trichogramma pretiosum $\quad 54$

Trifolium pratense $\quad 41$

Triticum aestivum 131, 147 
Vaccinium macrocarpon

Variabilité génétique

Venturia inaequalis

Verticillium dahliae

Vignobles

Vineyards

Vorlex Plus CP

White wood-rotting fungi

Wild cranberry
52,139

54,162

1

73

158

158

147

31

139 